\title{
Obstacles vs. Resources - Comparing the Effects of a Problem-Focused, Solution-Focused and Combined Approach on Perceived Goal Attainability and Commitment
}

\author{
Adam Abdulla ${ }^{1}$ (D) $\cdot$ Ruth Woods ${ }^{1}$
}

Accepted: 16 October 2020 / Published online: 11 November 2020

(C) The Author(s) 2020

\begin{abstract}
Previous research suggests that solution-focused (SF) questions may be superior to problem-focused (PF) alternatives for a range of practical and psychological outcomes. However, a great deal remains unknown regarding the effects of specific SF (or PF) approaches and the mechanisms through which they occur. The aim of this preregistered study was to investigate the extent to which SF questions targeting resources have a more positive effect on perceived goal attainability (PGA) and goal commitment than PF questions targeting obstacles or a combination of PF \& SF questions targeting both resources and obstacles. 115 students aged 15-16 were randomly assigned to either (i) a SF condition targeting resources, (ii) a PF condition targeting obstacles or (iii) a combined-approach condition targeting both. All participants were asked to identify a challenging area of study before answering condition-specific questions. Although not all statistically significant, results indicated that the SF group had higher mean PGA and goal commitment than both the PF and combined PF \& SF group. Effect size estimates were small-to-medium for PGA and small for goal commitment. Results of a mediation analysis suggested that condition had an indirect effect on goal commitment through enhanced PGA. Qualitative data analysis suggested that the PF question was more likely than the SF question to elicit thoughts of self-regulation, whereas the SF question was more likely to elicit thoughts of tools and resources. These findings are consistent with those from previous research and broaden our understanding of SF (vs PF) questions.
\end{abstract}

Keywords Solution-focused · Problem-focused $\cdot$ Perceived goal attainability · Goal commitment $\cdot$ Perceived obstacles $\cdot$ Perceived resources

Adam Abdulla

a.abdulla@rgu.ac.uk

School of Applied Social Studies, Robert Gordon University, Aberdeen, Scotland 


\section{Introduction}

\subsection{Background and Prior Research}

Solution-focused (SF) coaching and therapy are very much aligned with positive psychology. Rather than asking about weaknesses, obstacles or difficulties in goal attainment, SF coaches (and therapists) ask about strengths, resources and previous success (e.g. Iveson et al. 2012; Greene and Grant 2003; O'Connell et al. 2012). The SF approach was developed in the 1980s by family therapists including Steve de Shazer and Insoo Kim Berg who observed that focusing on "problems" was often ineffective (for more details see O'Connell and Palmer 2008). In the popular literature, SF approaches are often touted as superior to problem-focused (PF) alternatives for adults, children and adolescents (e.g. Franklin et al. 2018; Jackson and McKergow 2007; Taylor 2019).

Despite the wide appeal of SF coaching/therapy, it has been suggested that SF approaches are "more art than science" whereas positive psychology as a whole is "more science than art" (Bannink and Jackson 2011, p. 18). The SF approach has been most frequently investigated in the form of solution-focused brief therapy (SFBT). A number of meta-analyses and reviews of SFBT suggest that the approach is effective in various settings including health (Zhang et al. 2017) and education (Kim and Franklin 2009). Nevertheless, there are numerous problems with the existing evidence base. For example, Gingerich and Eisengart (2000) found that five well-controlled studies of SFBT reported significant benefits. However, the authors point out that four of those studies "did not compare SFBT with another psychotherapeutic intervention" and conclude that it is therefore impossible to determine whether the positive results "were due specifically to the SFBT intervention as opposed to general attention effects" ( $p$. 493). Had the studies compared SFBT with a problem-focused intervention, therefore, different results might have been obtained. Unfortunately, there have been only a handful of studies that explicitly compare SF and PF approaches.

For example, in a randomised controlled study Grant (2012) found that SF prompts and questions were more effective than PF alternatives in helping students generate action steps and (subjectively) approach their goals. Students asked SF questions also reported a statistically significant increase in positive affect and perceived self-efficacy as well as a statistically significant reduction in negative affect. PF questions, on the other hand, did not have a statistically significant effect on affect or perceived selfefficacy. Although Grant (2012) does not report effect sizes, these can be calculated from the data in his paper. Using Morris's (2007) formula for pretest-posttest control group designs, the estimated effect sizes are 0.44 for perceived self-efficacy, 0.21 for goal approach, 0.35 for positive affect and 0.24 for negative affect. According to Cohen (1988), these would all be considered small effects.

In a cross-cultural replication using the same procedure, variables and measures, Neipp et al. (2016) also found that SF questions were more effective than PF counterparts in enhancing perceived self-efficacy and goal approach and in reducing negative affect. Effect size estimates were again not provided by the authors but these can be calculated using their data. Like Grant (2012), Neipp and colleagues obtained small effects for all variables, according to Cohen's (1988) thresholds. Other studies further support the superiority of SF questions. For example, Braunstein and Grant (2016) 
found that relative to PF alternatives, SF questions led to a statistically significantly greater increase in positive affect, perceived self-efficacy and perceived goal progress and a statistically significantly greater reduction in negative affect, regardless of whether participants had approach or avoidance goals. Once again effect size estimates can be calculated using the data presented by the authors and Morris' (2007) recommendations. Estimated effect sizes were small for perceived goal progress $(d=0.26$ and $d=0.42$ ), small-to-medium for perceived self-efficacy $(d=0.44$ and $d=0.60)$, and medium-to-large for positive affect $(d=0.60$ and $d=0.80)$.

Finally, Grant and Gerrard (2019) once again found that SF questions were more effective than the PF alternatives in reducing negative affect and enhancing perceived goal attainment, self-efficacy and positive affect. In terms of enhancing perceived selfefficacy and reducing negative affect, SF questions were also more effective than a combined PF and SF approach. If Grant and Gerrard's (2019) SF condition is compared with the PF condition, effect size estimates are small-to-medium for perceived goal attainment $(d=0.48)$, large for perceived self-efficacy $(d=0.89)$, medium for positive affect $(d=0.68)$ and medium for negative affect $(d=0.66)$. If the SF condition is compared with the combined PF \& SF condition, effect size estimates are small for perceived goal attainment $(d=0.25)$, medium for perceived self-efficacy $(d=0.69)$, small for positive affect $(d=0.30)$ and medium for negative affect $(d=0.64)$.

The aforementioned studies collectively suggest that certain SF questions are more effective than PF alternatives in terms of enhancing various goal-related or affectrelated outcomes. Nevertheless, there are several limitations in the existing evidencebase. One limitation relates to the nature and number of questions. The studies discussed above all compared a battery of SF prompts and questions with a battery of PF alternatives. Table 1 illustrates this point by presenting the questions and prompts used in Grant's (2012) study. Very similar batteries of questions were used in the other studies mentioned above (e.g. Neipp et al. 2016). When all of these questions are included in a single experimental condition it is impossible to determine the effect of any one question (or prompt) in particular.

However, SF techniques and questions (like PF alternatives) come in many varieties. They include the "Miracle Question" (de Shazer 1988), scaling questions (e.g. Berg and Szabó 2005), questions about resources (e.g. Jackson and McKergow 2007), and questions about past success (e.g. Iveson et al. 2012). For both theoretical and practical reasons it would be helpful to examine these questions individually. In addition, there is the matter of ecological validity. There may be some doubt as to how frequently the questions in Table 1 are asked in "real life" or at least outside therapy and coaching.

Another limitation of previous studies is that the mechanisms of SF techniques have been largely unexplored. Whilst several different dependent variables were measured in the aforementioned studies (e.g. Grant 2012; Grant and Gerrard 2019; Neipp et al. 2016) the researchers did not examine mediators of effects. Indeed, mediational analyses are extremely rare in SF research (for an exception see Theeboom et al. 2016). Previous studies of SF (vs PF) questions have also included only quantitative analyses. A proper understanding of the mechanisms of SF techniques may also require analysis of qualitative data, i.e. a "mixed methods" approach. Responses in coaching/ therapy are almost invariably qualitative in nature. That is, individuals generally reveal their thoughts in words and sentences. These should be examined if we are truly to understand the effects of SF and PF questions. 
Table 1 Problem focused questions and solution-focused prompts/questions used in Grant (2012)

\begin{tabular}{|c|c|}
\hline Problem-focused Battery & Solution-focused Battery \\
\hline - "How long has this been a problem?" & $\begin{array}{l}\text { "Think about a possible solution to the problem you have just } \\
\text { described. Now, imagine the solution had somehow 'magically' } \\
\text { come about. Describe the solution" }\end{array}$ \\
\hline • "How did it start?" & $\begin{array}{l}\text { "Describe some ways you could start to move towards creating this } \\
\text { solution" }\end{array}$ \\
\hline $\begin{array}{l}\text { - "What are your thoughts about this } \\
\text { problem?" }\end{array}$ & • "What are your thoughts about this solution?" \\
\hline $\begin{array}{l}\text { - "How do you react when you have } \\
\text { those thoughts?" }\end{array}$ & • "How do you react when you have these thoughts?" \\
\hline $\begin{array}{l}\text { - "What impact is thinking about this } \\
\text { issue having on you?" }\end{array}$ & - "What impact is thinking about this solution having on you? \\
\hline
\end{tabular}

A fourth limitation in previous research is the absence of a measure of motivation or commitment. Speaking of SF coaching in a group context, O'Connell et al. (2012, p.105) write that "[t]he focus on solutions (not problems) and simple steps, and on utilisation of all the resources within the group, naturally builds energy levels and commitment to action" (italics added). Many other SF texts emphasise the importance of goal commitment and suggest that SF approaches may enhance it (e.g. Greene and Grant 2003; Jackson and McKergow 2007). However, previous studies of SF (vs. PF) questions have not measured effects on commitment.

The present study sought to address the limitations outlined above. Rather than employing a battery of SF/PF questions rarely asked in "real life", this study compared a single SF approach (focusing on resources) with a natural PF alternative (focusing on obstacles). In addition, a plausible mediational hypothesis was advanced and qualitative data were analysed to shed further light on potential mechanisms. Finally, goal commitment was included as a dependent variable. Each of these features is now explained.

\subsection{Perceived Goal Attainability and Goal Commitment}

In the present study the dependent variables were perceived goal attainability (PGA) and goal commitment. Participants were asked to identify an area of study that was proving challenging. The "goal" was to improve in that area. There are several reasons for focusing on PGA and goal commitment.

In Locke and Latham's (2013) edited book on goal-setting and task performance, an entire chapter is devoted to goal commitment (Klein et al. 2013). Meta-analyses assessing the relationship between goal commitment and task performance have found an average effect size of .23 (Klein et al. 2013). The positive effect of commitment on performance is supported by a large number of studies across a range of settings. Research indicates that high levels of commitment to educational goals are associated with several important benefits including greater academic adjustment (Germeijs and Verschueren 2007) and greater perseverance and effort (Tang et al. 2019). Higher levels of goal commitment have also been positively associated with enhanced strategy development (Earley et al. 1992) and positive affect and satisfaction with work (Roberson 1990). 
Perceived goal attainability (PGA) is another crucial variable for achievement and wellbeing. First, PGA is one of the main determinants of goal commitment (Klein et al. 2013). Several studies indicate that commitment to a goal declines as that goal becomes subjectively more difficult to attain (see Locke et al. 1988). Conversely, higher levels of PGA are associated with enhanced commitment, particularly in the early stages of goal pursuit (Huang et al. 2017). PGA also has associations with wellbeing, interacting with goal commitment. Brunstein (1993) found that when students were highly committed to personal goals, higher PGA was associated with greater subjective wellbeing. On the other hand, when students were highly committed to goals but considered those goals (almost) impossible to attain, their wellbeing was adversely affected. In addition, goal commitment significantly predicted goal progress only when PGA was high.

More recent studies corroborate Brunstein's (1993) findings. Boudrenghien et al. (2012) examined the effects of goal commitment and PGA in students who had received secondary school leaving qualifications. They found that "the positive effect of goal commitment on subjective well-being....disappeared or even changed direction when the educational goal was perceived as unattainable" (Boudrenghien et al. 2012, p.158). In their study of goals and mental health, Gamble et al. (2020) found that PGA was not only the strongest predictor of subsequent goal progress but also an extremely strong predictor of positive mental health and lower depressive symptoms.

In summary, research suggests that commitment and PGA are both extremely important for wellbeing and goal pursuit. Studies of SF (vs PF) approaches should therefore explore effects on both of these variables. Moreover, given that greater PGA is associated with greater commitment, any SF questions enhancing PGA may thereby also enhance commitment. In other words, the following mediational hypothesis should be investigated: SF (vs. PF) questions $\rightarrow$ enhanced PGA $\rightarrow$ enhanced commitment.

\subsection{Focusing on "Obstacles" - Solution-Focused vs. Problem-Focused Perspectives}

PF and SF approaches differ fundamentally in their treatment of "obstacles," making this an important dimension to explore. As already noted, SF approaches discourage a focus on obstacles. For example, Dierolf et al. (2009, p.32) suggest that "examining the obstacle is not important." Many other SF-oriented authors similarly argue that it is counterproductive to focus on obstacles (e.g. George and Ratner 2012; Jackson and McKergow 2007; Ratner and Yusuf 2015).

On the one hand, it might be thought that drawing attention to obstacles could lower PGA and commitment. As Ajzen (1991) notes, individuals can attend to only a limited number of beliefs at a given moment. According to the Theory of Planned Behaviour, therefore, whatever is most salient exerts the greatest influence on a person's attitudes. PF questions such as "What's preventing you from getting better grades?" may increase the salience of perceived obstacles ("I'm too easily distracted," "The textbook is terrible"). By making obstacles (temporarily) salient, PF questions may (temporarily) lower perceived behavioural control ("I can't study properly"), which may in turn lower PGA ("I'm unlikely to achieve better grades"). If asking students about obstacles lowers PGA, it may thereby undermine their commitment. Although this is only a theoretical possibility, research does suggest that questions that bias attention towards "negatives" subsequently affect people's judgements. For example, Lee et al. (2016) found that individuals (with deceased parents) reported lower life expectancy if they had just been asked whether their parents were still alive and (if not) at 
what age they had died. Similarly, students may report lower goal attainment expectancy if they have just been asked whether they are succeeding in achieving their goals and (if not) what is "holding them back."

On the other hand, drawing attention to obstacles may not lower PGA or goal commitment. For example, Artistico et al. (2013) examined the effect of a problem-solving session on individuals' PSE for exercise. The problem-solving session involved identifying daily obstacles to exercise and generating solutions to overcome them. Student participants assigned to the problem-solving condition reported greater increases in PSE for exercise than students assigned to the control groups. The authors do not report posttest PSE means for the control conditions, making it impossible to calculate effect sizes. However, they note that students in the problem-solving condition (focusing on obstacles) reported a mean increase in PSE of almost one standard deviation. Thus it seems that some PF approaches targeting obstacles may in fact enhance PSE, which is similar to PGA (Klein et al. 2013). In addition, research on "mental contrasting" indicates that focusing on obstacles can enhance commitment provided that individuals consider themselves capable of overcoming those obstacles (e.g. Oettingen et al. 2000, 2001, 2005).

In summary, there are reasons for thinking that focusing on obstacles may negatively bias judgements, which might subsequently reduce PGA and commitment. On the other hand, some research suggests that focusing on obstacles can (in some cases) enhance PSE and commitment. Whether PF questions about obstacles lower PGA and commitment is therefore an open question.

\subsection{Focusing on Resources - a Wholly Solution-Focused Approach}

Whereas SF practitioners tend to eschew "obstacles," they readily embrace talk of "resources." O'Connell et al. (2012, p.16) say that the SF coach "highlights and reinforces the coachee's strengths and resources". They also suggest that individuals should be encouraged to write down what resources they have as "[t]his process helps the development of self-efficacy" (O'Connell et al. 2012, p.16). Iveson et al. (2012, p.3) say that "[t]he essence of solution focused brief therapy, and solution focused coaching" is (amongst other things) "to look for resources rather than deficits."

Research very much supports the SF emphasis on resources. In a series of studies Schnelle et al. (2010) found that students who perceived themselves as having a large number of goal-relevant resources committed themselves to more approach-goals than students who perceived themselves as having fewer resources. In addition, in one of their studies outcome expectancy (almost identical to PGA) was found to mediate the effect of perceived resources on goal adoption. Students with greater resources had higher outcome expectancies (i.e. higher PGA), which apparently made them more likely to commit to approach-goals. In other words, there was evidence for the following causal chain: more resources $\rightarrow$ higher PGA $\rightarrow$ greater commitment. Other studies also suggest that the generation of goal-relevant resources raises commitment by raising PGA. In one such study participants were asked to list a number of means or resources that they believed would help them to achieve their goals (Kruglanski et al. 2011). The researchers found that the positive effect of the number of means or resources on goal commitment was mediated by both goal importance and the "perceived likelihood of goal attainment" (p.348). The latter variable is of course PGA. Studies such as these suggest that SF questions highlighting resources might enhance goal commitment by enhancing perceived goal attainability. 
Other research highlights the importance of perceived resources in motivating behaviour. Zhang and Gutierrez (2007) investigated the factors influencing use of information technology (IT). They found that perceived resources enhanced perceived behavioural control (PBC), which in turn led to stronger intentions to use IT. Kenny et al. (2003) explored the antecedents of high school students' engagement and vocational attitudes. They found that perceived support from family (an important resource) predicted greater commitment to school and higher expectations for achieving career goals (i.e. higher PGA). McWhirter et al. (1998) found that the more Mexican American high school girls perceived support from teachers and parents the more committed they were to future careers. Finally, in two classroom experiments Destin (2017) found that making young adolescents aware of financial resources enhanced their school motivation.

In summary, many studies suggest that helping students become aware of resources (e.g. supportive parents, helpful teachers etc.) may enhance both PGA and goal commitment. Research also suggests that the effect of perceived resources on goal commitment may be mediated by changes in PGA (e.g. Kruglanski et al. 2011; Schnelle et al. 2010). SF questions about resources may therefore strengthen commitment by enhancing PGA.

\subsection{Obstacles vs. Resources vs. Obstacles and Resources}

One of the putative advantages of the SF approach is that of brevity. Indeed, many SF commentators explicitly include the word "brief" when referring to SF coaching or therapy (e.g. Berg and Szabó 2005; Ratner and Yusuf 2015). They argue that by skipping "obstacle analysis" and focusing immediately on solutions, coaches can help individuals attain their goals more quickly (e.g. Dierolf et al. 2009). Some strictly solution-focused commentators argue that SFC is a stand-alone model that should never be combined with PF approaches, which only have an undermining effect (e.g. Ratner and Yusuf 2015). If these intuitions are correct, then a SF approach should be more effective than both a PF and combined PF \& SF approach. Grant and Gerrard (2019) found some support for this hypothesis: in terms of enhancing perceived selfefficacy (PSE) and reducing negative affect, SF questions were more effective than (i) PF questions alone and (ii) a combination of PF and SF questions.

\section{The Present Study}

The present study compared the effects of a PF approach targeting obstacles, a SF approach targeting resources, and a combined PF \& SF targeting both obstacles and resources on perceived goal attainability and goal commitment. More specifically, the present study was designed to shed light on the following question: to what extent is a SF approach targeting resources more effective than (i) a PF approach targeting obstacles and (ii) a combined PF \& SF approach targeting both obstacles and resources? An opportunity sample of students from a UK school were recruited to participate. Amongst educational psychologists in the UK, solution-focused approaches have been popular for many years (e.g. Stobie et al. 2005). A school in the UK therefore seemed to be an excellent location for the current investigation. 
The following hypotheses were investigated in the present study:

H1 (PGA):

Students asked to identify and think of ways to use resources experience greater perceived goal attainability (PGA) than (a) students asked to identify and think of ways to overcome obstacles, and (b) students asked both to identify and think of ways to overcome obstacles and to identify and think of ways to use resources.

\section{H2 (Goal commitment):}

Students asked to identify and think of ways to use resources experience greater goal commitment than (a) students asked to identify and think of ways to overcome obstacles and (b) students asked both to identify and think of ways to overcome obstacles and to identify and think of ways to use resources.

PGA is itself a major determinant of goal commitment. Thus the following hypothesis was also advanced:

H3: PGA is positively associated with goal commitment.

Finally, a mediational hypothesis was investigated. If SF questions about resources enhance PGA, and PGA is positively associated with commitment, then SF questions about resources might enhance commitment via enhanced PGA. Moreover, previous research does indeed suggest that the positive effect of perceived resources on goal commitment is mediated by enhanced PGA (e.g. Kruglanski et al. 2011; Schnelle et al. 2010). The following hypothesis was therefore also investigated:

H4: The effect of condition on goal commitment (see $\mathrm{H} 2$ above) is mediated by altered PGA.

The hypotheses above are expressed in the all-or-nothing (effect or no effect) language associated with null hypothesis significance testing (NHST). There are good reasons, however, to adopt a more nuanced approach, namely "estimation thinking" (e.g. Cumming 2012). Adopting this approach means asking not "is there an effect?" but rather "how large is the effect likely to be, given the data obtained?" In order to answer the latter question, more attention is paid to effect sizes and confidence intervals than to $p$-values and NHST.

\section{Method}

\subsection{Participants}

115 female students aged $15-16(\mathrm{M}=16.02 ; \mathrm{SD}=0.44)$ participated. Students attended an independent all-female secondary school in London. All participants gave informed consent. No participants opted out. The study was approved by the School of Applied Social Studies Ethics Committee at Robert Gordon University in Aberdeen, Scotland 
(RGU). The largest possible sample size (within the given school) was recruited in order to increase the accuracy of estimated effect sizes.

\subsection{Procedure}

Participants were randomly assigned to either the PF condition targeting obstacles $(n=$ $39)$, the SF condition targeting resources $(n=38)$, or the combined PF \& SF condition targeting both obstacles and resources $(n=38)$. Students in each condition were sent a link to the corresponding survey, which they completed through Google Forms. Participants were asked to complete their surveys during a non-teaching slot between lessons. After reading the participation information and giving consent, students were asked to identify an area of study in which their performance was (to them) unsatisfactory. Subsequent questions differed according to condition. In order to control for the number of ideas generated participants in each condition were asked to list 1-2 obstacles/resources. In all conditions, the goal was presented as "improving" in the area they had identified.

In the PF condition, students were initially given the following prompt: "Please list 1-2 things that (might) hold you back in the area you identified." They were then provided with space to list their obstacles. The next prompt was as follows: "Think about how you could overcome the things you just listed. What could you do? Please list 1-2 things." Once again, they were provided with some space to write down their ideas. This two-step PF approach (i.e. 1) identifying obstacles and then 2) thinking of ways to overcome them) is widely recommended in the personal development literature (e.g, Bowkett and Percival 2011; Canfield and Chee 2013; Kets de Vries 2006; Madrid and Quick 2007). It is also commonly endorsed in texts about students (e.g. Mazza et al. 2016; Wolraich 2008).

The layout in the SF condition was identical except that the two prompts/questions were: "Please list 1-2 things that (might) help you in the area you identified" and "Think about how you could use the things you just listed. What could you do? Please list 1-2 things." Thus students in the PF and SF conditions were both asked a pair of questions. The first question in the pair asked students to identify obstacles/resources, and the second question asked students how they might overcome/use those obstacles/ resources. In the combined PF \& SF condition, participants answered first the PF pair (concerning obstacles) and then the SF pair (concerning resources).

After answering condition-specific questions, all participants were presented with the questions for the dependent measures (i.e. PGA and commitment). Participants were given up to 10 min to complete their surveys and were told that they could stop at any point. When all participants had submitted their answers they were debriefed as to the purpose of the study.

\section{Measures}

\subsection{Perceived Goal Attainability (PGA)}

This was assessed using a three-item measure derived from Huang et al. (2017). For each item participants were asked to give a number between 0 and 10 (e.g. "On a scale 
from 0 to 10 , how likely is it that you will improve in this area?'). Higher scores indicated higher PGA. Internal consistency was high $(\alpha=0.83)$.

\subsection{Goal Commitment}

This was measured using the four-item KUT commitment measure developed by Klein and colleagues (see Klein et al. 2014). For each item, a 7-point response scale was used (e.g. "On a scale from 1 to 7, how committed are you to improving in this area?"). Higher scores indicated higher goal commitment. Internal consistency was extremely high $(\alpha=0.91)$.

\subsection{Analytical Strategy}

In order to examine the effect of condition on PGA and commitment, two ANOVAs were conducted - one for each variable. Although PGA is a determinant of commitment, the two variables are conceptually distinct. The interest in the present study lay in the effects of condition on each variable separately (and a subsequent mediation analysis). There was therefore no interest in a linear composite of the variables. In such a situation, separate ANOVAs (rather than a single MANOVA) are appropriate (Huberty and Morris 1989).

Alpha was set at 0.05 for each test. Although some commentators recommend a Bonferroni correction when more than one ANOVA is performed, this can severely reduce the power required to detect important effects (e.g. Gelman et al. 2012). Readers may of course apply their own alpha adjustment. As already indicated, however, in the present study more attention was paid to effect sizes and confidence intervals than to $p$ values and NHST. Estimated effect sizes were calculated using Cohen's $d$.

In order to investigate whether any effect of condition on commitment was mediated by PGA, the approach originally recommended by Hayes and Preacher (2014) was applied, using Hayes' PROCESS macro. Two relative indirect effects were calculated, one for the influence of the SF condition relative to the PF condition $\left(a_{1} b\right)$, and one for the influence of the SF condition relative to the $\mathrm{PF} \& \mathrm{SF}$ condition $\left(a_{2} b\right)$. According to $\mathrm{H} 4$, the effect of condition on goal commitment is mediated by changes in PGA. In the present analysis the SF condition was coded as the reference group. The PF and PF \& SF groups were expected to have lower commitment than the SF group as a result of reduced PGA. Negative relative indirect effects were therefore anticipated.

Following the statistical tests, qualitative data were coded and analysed in order to see whether they might help to explain the quantitative findings. Specifically, we wondered whether PF and SF questions might elicit different types of thoughts, which might influence PGA and commitment. Analysis of students' written responses might therefore clarify how or why SF and PF questions have differential effects on the dependent variables.

The assumptions for ANOVA and multiple regression (e.g. normality, homoscedasticity) appeared to have been met in all cases. One extreme score was identified in the $\mathrm{PF}$ group. Analyses were conducted both with and without this outlier to test for any differences in results. 


\section{Results}

All students completed the surveys and there were no missing data. Group means and standard deviations for PGA and goal commitment are displayed in Table 2.

\subsection{The Effect of Condition on Perceived Goal Attainability}

A one-way between-subjects ANOVA was conducted with condition as the independent variable and PGA as the dependent variable. With the outlier included, the effect of condition on PGA was on the borderline of statistical significance: $F(2,112)=2.90$, $p=.059, \eta^{2}=.05$. Planned comparisons using Fisher's LSD indicated that the PGA mean of the SF condition was statistically significantly higher than that of the PF condition $(p=.04)$ and combined PF \& SF condition $(p=.04)$. If Cohen's (1988) criteria are applied, the estimated effect of the SF intervention on PGA was small-tomedium when compared with either the PF condition $(d=0.48,95 \%$ CI $[0.026,0.933])$ or PF \& SF condition $(d=0.51,[0.051,0.965])$.

When the outlier was removed, the $p$ value for the overall ANOVA was raised but the effect of condition on PGA was still close to statistical significance: $F(2,111)=$ $2.71, p=.07, \eta^{2}=.05$. Planned comparisons using Fisher's LSD indicated that the PGA mean of the SF group was still statistically significantly higher than that of the combined PF \& SF group $(p=.03)$. However, the difference between the PGA mean for the SF group and the new PGA mean for the PF group $(M=5.59)$ was now just above the threshold for statistical significance $(p=.08)$. If Cohen's (1988) criteria are applied, the estimated effect of the SF intervention when compared with the PF condition might now be described as small rather than small-to-medium but the difference was only slight $(d=0.43[-0.031,0.879])$.

\subsection{The Effect of Condition on Goal Commitment}

A one-way between-subjects ANOVA was conducted with condition as the independent variable and goal commitment as the dependent variable. Effect size estimates were scarcely affected by the outlier and NHST conclusions were identical in each case. Only the analysis excluding the outlier is reported.

The effect of condition on goal commitment was not statistically significant: $F(2$, $111)=0.80, p=.45, \eta^{2}=.01$. However, mean goal commitment was higher in the SF

Table 2 Means and standard deviations for perceived goal attainability (PGA) and goal commitment for the three conditions

\begin{tabular}{llll}
\hline & $\begin{array}{l}\text { PF Group } \\
(n=39)\end{array}$ & $\begin{array}{l}\text { SF Group } \\
(n=38)\end{array}$ & $\begin{array}{l}\text { PF \& SF Group } \\
(n=38)\end{array}$ \\
\hline Perceived Goal Attainability (PGA) & $\begin{array}{l}5.47 \\
(\mathrm{SD}=1.58)\end{array}$ & $\begin{array}{l}6.15 \\
(\mathrm{SD}=1.22)\end{array}$ & $\begin{array}{l}5.46 \\
(\mathrm{SD}=1.46) \\
\text { Goal Commitment }\end{array}$ \\
$\begin{array}{l}4.75 \\
(\mathrm{SD}=1.39)\end{array}$ & $\begin{array}{l}5.16 \\
(\mathrm{SD}=1.16)\end{array}$ & $\begin{array}{l}4.84 \\
(\mathrm{SD}=1.31)\end{array}$ \\
\hline
\end{tabular}

$\mathrm{SD}=$ standard deviation 
group $(\mathrm{M}=5.16)$ than in the PF $(\mathrm{M}=4.85)$ or $\mathrm{PF} \& \mathrm{SF}(\mathrm{M}=4.84)$ groups. When compared with either the PF or PF \& SF condition the estimated effect of the SF condition was small $(d=0.26,[-0.197,0.706])$.

\subsection{The Association between Perceived Goal Attainability and Goal Commitment (Controlling for Condition)}

Multiple regression was used to investigate the association between PGA and goal commitment (controlling for condition). Two dummy variables were created to code the three conditions. Goal commitment was then regressed on PGA and the two dummy variables. The overall regression was statistically significant: $F(3,110)=8.22, p<.001, R^{2}=.18$. The association between PGA and goal commitment was also statistically significant: $b=.38$, $[.22, .53], t=4.77, p<.001$. The standardised beta coefficient was .42 .

\subsection{The Indirect Effect of Condition on Goal Commitment through Perceived Goal Attainability}

The first relative indirect effect $\left(a_{1} b\right)$ was estimated as -0.2113 , which suggests that when compared with the SF condition the PF condition lowered commitment by 0.2113 units as a result of reducing PGA (which in turn affected commitment). A 95\% bootstrap confidence interval (CI) for this indirect effect based on 5000 bootstrap samples was entirely negative $[-0.4721,-0.0008]$, implying that the indirect effect was statistically different from zero.

The second relative indirect effect $\left(a_{2} b\right)$ was estimated as -0.2576 , which suggests that when compared with the SF condition, the combined PF \& SF condition lowered commitment by 0.2576 units as a result of reducing PGA (which in turn affected commitment). A $95 \%$ bootstrap confidence interval for this indirect effect based on 5000 bootstrap samples was entirely negative $[-0.5891,-0.0240]$. Since both relative indirect effects were statistically different from zero, it is assumed that there was good evidence for mediation (Hayes 2018). That is to say, condition appeared to have an indirect effect on commitment through PGA. However, the partially standardised effect sizes were -0.1702 and -0.2075 , suggesting fairly small effects.

Estimates for the two relative direct effects $\left(c_{1}\right.$ and $\left.c_{2}\right)$ were also obtained from the regression output. Both estimates were extremely small and confidence intervals were wide and included zero: $c_{1}=0.098[-0.427,0.622]$ and $c_{2}=0.040[-0.478,0.557]$. The $p$-values were also far from statistical significance $(p=.71$ and $p=.88$, respectively). In addition, the omnibus test of the direct effect was not statistically significant $(p=.93)$. There was therefore no good evidence to suggest that condition had a (meaningful) direct effect on commitment (independent of PGA).

\subsection{Analysis of Qualitative Data}

The quantitative analysis reported above suggested that condition had a small-tomedium effect on PGA and a small effect on commitment (through altered PGA). It was suspected that the PF and SF questions elicited different types of thoughts, which might help to explain group differences on the dependent variables. Students' qualitative responses were therefore analysed in order to explore this possibility. 
Students in the SF group were not expected to identify any "obstacles" to goal attainment since no questions in their condition concerned obstacles. However, students in all conditions were asked at least one question that could have elicited "solutions." Note that even PF students were asked such a question since the second question in the PF pair asked how they might overcome their obstacles. Attention was therefore focused on what students wrote in answer to the second question in the PF/SF pair. Answers were analysed in terms of suggested solutions. A "solution" was defined as any proposed measure that might facilitate goal attainment.

In the first stage of the analysis (conducted by the first author), all student comments were coded for solutions regardless of condition. At this stage the approach was predominantly inductive as codes were largely suggested by the data themselves. For example, "do more practice" was coded as "practice." There were 222 comments in total, which resulted in a large number of codes. In the second stage of the analysis, similar or related codes were merged, following discussion between the first and second authors. For example, "practice" and "revision" became "Practice/Revision." In addition, codes with a similar theme were subsumed under one overarching code. For example, "meet with my teacher," "asking friends for advice" and "talking with Spanish people" were all coded as "social support."

The two authors eventually established a set of 6 codes: 1) Practice/Revision, 2) Self-regulation, 3) Social Support, 4) Resources and Tools, 5) Personal Notes, 6) Unusual Approach. The last category was used for proposed solutions that did not fit into any of the other categories. In establishing, naming and defining this highest level of codes the authors drew on their knowledge of the literature and on certain predefined concepts. For example, the term "selfregulation" was proposed to cover comments such as "concentrate more in lessons," and "being more motivated." Thus a top-down approach was applied (in some instances) at this stage. Following construction of a coding scheme (which provided guidelines for use of each code), the two co-authors independently coded the entire set of comments, applying one of the 6 codes to each student comment. Inter-rater agreement was high (Cohen's $\kappa=0.85, p<.001$ ).

Table 3 reveals the number of times each type of solution was proposed within each group. Comments within the PF\&SF condition were divided into comments made in response to the PF question (about obstacles) and comments made in response to the SF question (about resources). The numbers reflect the first author's coding but the second author's was almost identical.

Inter-rater agreement was low for "Unusual Approach" and so this category was disregarded. Table 3 suggested two potentially meaningful between-question differences. First, it appeared that the PF question evoked more thought of "Self-regulation" (SR) than the SF question. When the PF condition was compared with the SF condition, it was observed that SR was mentioned almost three times more often in the former than in the later (22 vs 8). Similarly, when the PF and SF questions were isolated within the PF\&SF condition, it was observed that SR was mentioned twice as frequently following the former (14 vs 7). For "Resources \& Tools" (R\&T), the pattern was reversed. There were almost twice as many mentions of R\&T in the SF condition as there were in the PF condition (10 vs 6). Similarly, within the PF\&SF condition, R\&T occurred almost three times more often following the SF question than the PF question (14 vs 5). 
Table 3 The number of times each solution type was proposed in each experimental condition

\begin{tabular}{|c|c|c|c|c|}
\hline & \multirow[b]{2}{*}{$\mathrm{PF}$} & \multirow[b]{2}{*}{$\mathrm{SF}$} & \multicolumn{2}{|c|}{ PF\&SF } \\
\hline & & & $\mathrm{PF}$ & SF \\
\hline Practice/Revision & 18 & 23 & 17 & 10 \\
\hline Self-regulation & 22 & 8 & 14 & 7 \\
\hline Social Support & 10 & 8 & 8 & 14 \\
\hline Resources \& Tools & 6 & 10 & 5 & 14 \\
\hline Personal Notes & 3 & 2 & 1 & 1 \\
\hline Unusual Approach & 0 & 9 & 5 & 6 \\
\hline
\end{tabular}

In order to arrive at a more accurate estimate of each question's tendency to elicit particular "solutions", totals were calculated for the PF and SF questions, collapsing across conditions. That is, the numbers in the first and third columns of Table 3 were added together as were the numbers in the second and fourth. "Unusual Approach" was omitted due to low inter-rater agreement. Table 4 displays the resulting totals.

The data in Table 4 were submitted to a chi-square test of independence. There was a statistically significant association between type of question (PF/SF) and type of solution: $\chi^{2}(4)=13.85, p<0.08$. Examination of adjusted residuals within the Selfregulation (SR) and Resources \& Tools $(\mathrm{R} \& \mathrm{~T})$ cells revealed that the differences in response frequencies for SR and $R \& T$ were statistically significant $(p<0.05)$. Calculation of odds ratios revealed that the odds of obtaining a self-regulation solution were 2.89 times greater if students were asked the PF rather than the SF question. Conversely, the odds of obtaining a Resources \& Tools solution were 2.78 greater if students were asked the SF rather than the PF question.

\section{Discussion}

The primary aim of the present study was to investigate the extent to which SF questions addressing resources have a more positive effect on perceived goal attainability and goal commitment than PF questions addressing obstacles (or a combination of PF and SF questions addressing both). In addition, the study sought to shed light on the mechanisms through which effects may occur. In what follows, more attention is paid to effect sizes and confidence intervals than to $p$-values, since the latter can be extremely volatile and unreliable (e.g. Cumming 2008).

Table 4 The number of times each solution type was proposed following each type of question

\begin{tabular}{lll}
\hline & PF & SF \\
\hline Practice/Revision & 35 & 33 \\
Self-regulation & 36 & 15 \\
Social Support & 18 & 22 \\
Resources \& Tools & 11 & 24 \\
Personal Notes & 4 & 3 \\
\hline
\end{tabular}


As predicted by H1(a), H1(b) and solution-focused thinking, PGA was higher in the SF condition than in the PF and PF \& SF conditions. After removal of an outlier, the estimated effect of the SF condition was small-to-medium when compared with the PF condition $(d=0.43,[-0.031,0.879])$ and of medium size when compared with the PF $\&$ SF condition $(d=0.51,[0.051,0.965])$. Admittedly, the CIs are wide and range from very small (or even slightly negative) to fairly large. This highlights the needs for replications - with precision in planning - and ultimately a meta-analysis (Cumming 2012). However, the most plausible (point) estimates are in the small-to-medium range. In practical terms, this suggests that when students have identified an unsatisfactory area of study, asking them about resources rather than obstacles (or resources and obstacles) may have a somewhat positive impact on the extent to which they believe they can improve in that area. Given the benefits of PGA for both wellbeing and goal pursuit (e.g. Boudrenghien et al. 2012; Gamble et al. 2020; Huang et al. 2017), this may be an important finding.

The small-to-medium effect on PGA is also consistent with previous research. At the beginning of this paper, effect size estimates were calculated for other studies of PF/SF questions. The formula recommended by Morris (2007) for pretest-posttest control group designs was used. If estimates are recalculated on the basis of posttest data alone (to make them compatible with the present study), the similarities in findings remain. For example, in Cohen's (1988) terms, Neipp et al. (2016) found that relative to PF questions SF questions had small positive effects on perceived self-efficacy (PSE) and (perceived) goal approach. PSE and (perceived) goal approach are close to PGA as measured in the present study. Similarly, Grant (2012) found that relative to PF questions, SF questions had a small positive effect on PSE and a small-to-medium effect on perceived goal approach. Finally, Grant and Gerrard (2019) found that relative to either PF questions or PF \& SF questions, SF questions (alone) had a small positive effect on perceived goal attainment and a medium-sized effect on perceived selfefficacy. Thus the findings of the present study are consistent with prior research.

However, the present study also broadens our understanding. Whereas previous studies had used a whole battery of PF and SF questions, the present study narrowed the focus to a single dimension: obstacles versus resources. The apparent superiority of the SF approach was observed even in this narrower contrast. Moreover, unlike previous studies (which included elaborate prompts not normally used outside coaching/therapy), the present study compared simpler and more "natural" questions. Thus it would appear that the advantage of the SF approach may extend to everyday contexts.

It would be reasonable to ask why the SF condition had higher mean PGA than the other two conditions. Of course sampling error remains a possibility. However, the likelihood of that explanation is undermined by the consistency of the present results with previous research. Moreover, analysis of qualitative data suggests that the PF and SF question may have elicited different types of thoughts, which may in turn have affected PGA.

The PF question was much more likely to elicit thoughts of self-regulation than the SF question. In addition, the SF question was much more likely to elicit thoughts of resources and tools. The latter finding was not surprising given that the SF question explicitly asked about resources. However, the former finding (concerning selfregulation) was not anticipated and would need to be replicated in future studies. Nevertheless, the combination of these findings may help to explain group differences 
in PGA. The "self-regulation" code was defined so as to include time management, concentration in class, self-motivation, ignoring distractions and the exercise of selfdiscipline. The definition was based on widely accepted views of self-regulation in learning (e.g. Usher and Pajares 2008). Research suggests that perceived self-efficacy for self-regulation declines throughout high school and adolescence (e.g. Caprara et al. 2008). Students who are reminded of self-regulation issues may come to doubt whether they can attain their goals. Specifically, PF questions targeting obstacles may draw attention to self-regulation failures, which may in turn lower PGA. On the other hand, as noted, the SF question in the present study appeared to elicit more thoughts of tools and resources (i.e. external solutions) than the PF question. The perception of goalrelevant resources is associated with higher PGA (e.g. Schnelle et al. 2010). Relative to the PF group, therefore, the SF group may have benefitted from (largely) bypassing self-regulation issues and focusing on resources and tools. Although the PF\&SF group would also have had the "benefit" of the question about resources, thoughts of selfregulation (evoked by the PF question) may have outweighed or undermined that benefit.

The present study also introduced a new variable into research on PF and SF questions - goal commitment. On the basis of SF thinking it was hypothesised that relative to PF and PF \& SF questions, SF questions (alone) have a positive effect on goal commitment ( $\mathrm{H} 2 \mathrm{a}$ and $\mathrm{H} 2 \mathrm{~b})$. Moreover, it was hypothesised that this effect is mediated by altered PGA (H4), assuming that PGA and goal commitment are related (H3). Evidence was indeed found for a positive association between PGA and goal commitment. Although the effect of condition on goal commitment was not statistically significant, mean commitment was indeed slightly higher in the SF group than in the PF or PF \& SF groups. In addition, results of the mediation analysis suggested that the effect of condition on commitment is indeed mediated by enhanced PGA. Thus if questions about (i) obstacles, (ii) resources and (iii) obstacles and resources do have differing effects on goal commitment, then PGA may be a likely mechanism.

\section{Conclusion}

Previous studies suggest that compared to PF questions (as a whole) SF questions (as a whole) may have positive effects on variables such as perceived self-efficacy and perceived goal approach (e.g. Grant 2012; Neipp et al. 2016). This study builds on and extends previous research by conducting a narrower and more ecologically valid comparison: questions about obstacles vs. questions about resources. Individuals are frequently asked about barriers to goal attainment (e.g. "What's holding you back?"). Alternatively, they may be asked about resources that facilitate goal attainment (e.g. "What could help you move forward?"). The results of this study suggest that the latter type of question may be somewhat more effective in making goals appear attainable and raising commitment to attaining them. Effects on PGA apparently approach a medium-size whilst those on commitment are probably small.

It should not be concluded from this study that attending to obstacles is invariably counterproductive. Research on mental contrasting and implementation intentions (MCII) has shown that reflecting on obstacles can facilitate goal pursuit provided that individuals (i) have previously imagined the benefits of goal attainment, (ii) have faith 
in their ability to overcome the obstacles, and (iii) make specific plans to do so (e.g. Oettingen and Gollwitzer 2010). Future studies could therefore pit a solution-focused approach against MCII.

Like all studies, the present study has its limitations. The absence of baseline measures or a neutral control group makes it impossible to determine whether the SF condition raised PGA (and commitment) or whether the PF and PF $+\mathrm{SF}$ condition lowered it (or both). Researchers seeking to replicate this study may wish to include a neutral control group or measure variables both before and after the intervention. In addition, future research should investigate potential moderators. For example, it might be the case that individuals with a high sense of self-efficacy are motivated by the perception of obstacles whilst individuals with lower perceived self-efficacy are discouraged by it. Researchers may also wish to test whether PF questions about obstacles do indeed lower students' perceived self-efficacy for self-regulation (SR) and, if so, whether this mediates the effect of the PF question on PGA.

It is also important to consider the generalisability of the findings. The most conservative approach would be to limit the population about which generalisations are made to female UK secondary school students aged 15-16. According to UK government figures, there were over 420,000 such students in 2019 (Department for Education 2019). Thus even if an extremely conservative approach is adopted, the findings of this study could be applied to almost half a million individuals. In reality however, there are good reasons to assume that they extend much further than this. As already observed, the results reported here are highly consistent with those of previous studies which involved older participants (male and female) and different nationalities (e.g. Grant and Gerrard 2019; Neipp et al. 2016). The effect of SF questions may therefore be quite similar across students of different ages and genders. However future studies will need to explore whether gender and age moderate effects.

In conclusion, (as far as we are aware) this is the first study to compare the effects of a single SF approach ("resources") against a single PF alternative ("obstacles"). Since its inception, positive psychology has focused on what people have rather than what they lack, on what they may use rather than what they must "repair" (e.g. Seligman and Csikszentmihalyi 2000). Adopting this philosophy, the present study suggests that an approach based on resources may be more effective than one based on obstacles in terms of increasing expectations of goal attainment and (thereby) enhancing goal commitment. If these results can be replicated, this would constitute a very important finding within applied positive psychology.

Acknowledgments The authors would like to acknowledge the help of Kevin Hallgren, Kilem Gwet and Donald Sharpe who provided extremely useful feedback on the qualitative data analysis.

Data Availability Data are available on request from the first author.

\section{Compliance with Ethical Standards}

Conflict of Interest/Competing Interests On behalf of all authors, the corresponding author states that there is no conflict of interest.

Code Availability Not applicable. 
Open Access This article is licensed under a Creative Commons Attribution 4.0 International License, which permits use, sharing, adaptation, distribution and reproduction in any medium or format, as long as you give appropriate credit to the original author(s) and the source, provide a link to the Creative Commons licence, and indicate if changes were made. The images or other third party material in this article are included in the article's Creative Commons licence, unless indicated otherwise in a credit line to the material. If material is not included in the article's Creative Commons licence and your intended use is not permitted by statutory regulation or exceeds the permitted use, you will need to obtain permission directly from the copyright holder. To view a copy of this licence, visit http://creativecommons.org/licenses/by/4.0/.

\section{References}

Ajzen, I. (1991). The theory of planned behaviour. Organizational Behaviour and Human Decision Processes, 50, 179-211.

Artistico, D., Pinto, A. M., Douek, J., Black, J., \& Pezzuti, L. (2013). The value of removing daily obstacles via everyday problem-solving theory: Developing an applied novel procedure to increase self-efficacy for exercise. Frontiers in Psychology, 4. https://doi.org/10.3389/fpsyg.2013.00020.

Bannink, F. P., \& Jackson, P. Z. (2011). Positive psychology and solution focus - Looking at similarities and differences. InterAction The Journal of Solution Focus in Organisations, 3(1), 8-20.

Berg, I. K., \& Szabó, P. (2005). Brief coaching for lasting solutions. New York: Norton.

Boudrenghien, G., Frenay, M., \& Bourgeois, É. (2012). Unattainable educational goals: Disengagement, reengagement with alternative goals, and consequences for subjective well-being. European Review of Applied Psychology, 62, 147-159 ezproxy.rgu.ac.uk/10.1016/j.erap.2012.04.002.

Bowkett, S., \& Percival, S. (2011). Coaching emotional intelligence in the classroom. Hove: Routledge.

Braunstein, K., \& Grant, A. M. (2016). Approaching solutions or avoiding problems? The differential effects of approach and avoidance goals with solution-focused and problem-focused coaching questions. Coaching: An International Journal of Theory, Research and Practice, 9(2), 93-109.

Brunstein, J. C. (1993). Personal goals and subjective well-being: A longitudinal study. Journal of Personality and Social Psychol, 65, 1061-1070.

Canfield, J., \& Chee, P. (2013). Coaching for breakthrough success: Proven techniques for making impossible dreams possible. New York McGraw-Hill Education.

Caprara, G. V., Fida, R., Vecchione, M., Del Bove, G., Vecchio, G. M., Barbaranelli, C., \& Bandura, A. (2008). Longitudinal analysis of the role of perceived self-efficacy for self-regulated learning in academic continuance and achievement. Journal of Educational Psychology, 100, 525-534.

Cohen, J. (1988). Statistical power analysis for the behavioral sciences (2nd ed.). Hillsdale: Lawrence Earlbaum Associates.

Cumming, G. (2008). Replication and p intervals: P values predict the future only vaguely, but confidence intervals do much better. Perspectives on Psychological Science, 3(4), 286-300.

Cumming, G. (2012). Understanding the new statistics: Effect sizes, confidence intervals, and meta-analysis. New York: Routledge.

de Shazer, S. (1988). Clues: Investigating solutions in brief therapy. New York: Norton.

Department for Education. (2019). Schools, pupils and their characteristics: January 2019. Retrieved from https:/www.gov.uk/government/statistics/schools-pupils-and-their-characteristics-january-2019

Destin, M. (2017). An open path to the future: Perceived financial resources and school motivation. Journal of Early Adolescence, 37, 1004-1031.

Dierolf, K., Meier, D., \& Szabo, P. (2009). Coaching Plain \& Simple: Solution-focused brief coaching essentials. New York: W.W. Norton \& Co..

Earley, P. C., Shalley, C. E., \& Northcraft, G. B. (1992). I think I can, I think I can...processing time and strategy effects of goal acceptance/rejection decisions. Organizational Behavior and Human Decision Processes, 53, 1-13.

Franklin, C., Streeter, C. L., Webb, L., \& Guz, S. (2018). Solution focused brief therapy in alternative schools. New York: Routledge.

Gamble, B., Tippett, L. J., Moreau, D., \& Addis, D. R. (2020). The futures we want: How goal-directed imagination relates to mental health. [PDF file]. Retrieved from: https://bc8c6d88-a2e4-417e-a6cc72d924b295cf.filesusr.com/ugd/aaa34a_1ea4acc76c30481eb366a961c778783f.pdf . 
Gelman, A., Hill, J., \& Yajima, M. (2012). Why we (usually) don't have to worry about multiple comparisons. Journal of Research on Educational Effectiveness, 5, 189-211.

Germeijs, V., \& Verschueren, K. (2007). High school students' career decision-making process: Consequences for choice implementation in higher education. Journal of Vocational Behavior, 70, 223-241.

Gingerich, W. J., \& Eisengart, S. (2000). Solution-focused brief therapy: A review of the outcome research. Family Process, 39(4), 477-498.

Grant, A. (2012). Making positive change: A randomized study comparing solution-focused vs. problemfocused coaching questions. Journal of Systemic Therapies, 31(2), 21-35.

Grant, A. M., \& Gerrard, B. (2019). Comparing problem-focused, solution-focused and combined problemfocused/solution-focused coaching approach: Solution-focused coaching questions mitigate the negative impact of dysfunctional attitudes. Coaching: An International Journal of Theory, Research and Practice, 1-17. https://doi.org/10.1080/17521882.2019.1599030.

Greene, J., \& Grant, A. M. (2003). Solution-focused coaching. Harlow: Pearson Education.

Hayes, A. F. (2018). Introduction to mediation, moderation, and conditional process analysis (2nd ed.). New York: The Guilford Press.

Hayes, A. F., \& Preacher, K. J. (2014). Statistical mediation analysis with a multicategorical independent variable. British Journal of Mathematical and Statistical Psychology, 67(3), 451-470. https://doi.org/10. 1111/bmsp.12028.

Huang, S.-C., Jin, L., \& Zhang, Y. (2017). Step by step: Sub-goals as a source of motivation. Organ Behav Human Decision Processes, 141, 1-15. https://doi.org/10.1016/j.obhdp.2017.05.001.

Huberty, C. J., \& Morris, J. D. (1989). Multivariate analysis versus multiple univariate analyses. Psychological Bulletin, 105, 302-308.

Iveson, C., George, E., \& Ratner, H. (2012). Brief coaching: A solution focused approach. London: Routledge.

Jackson, P. Z., \& McKergow, M. (2007). The solutions focus: Making coaching and change simple. London: Nicholas Brealey Publishing.

Kenny, M. E., Blustein, D. L., Chaves, A., Grossman, J. M., \& Gallagher, L. A. (2003). The role of perceived barriers and relational support in the educational and vocational lives of urban high school students. Journal of Counseling Psychology, 50(2), 142-155.

Kets de Vries, M. F. R. (2006). The leadership mystique (2nd ed.). New York: Prentice Hall.

Kim, J. S., \& Franklin, C. (2009). Solution focused brief therapy in schools: A review of the outcome literature. Children and Youth Services. 31, 464-470.

Klein, H. J., Cooper, J. T., Molloy, J. C., \& Swanson, J. A. (2014). The assessment of commitment: Advantages of a unidimensional, target-free approach. Journal of Applied Psychology, 99, 222-238.

Klein, H. J., Cooper, J. T., \& Monahan, C. A. (2013). Goal commitment. In E. A. Locke \& G. P. Latham (Eds.), New developments in goal setting and task performance (pp. 65-89). New York: Routledge.

Kruglanski, A. W., Pierro, A., \& Sheveland, A. (2011). How many roads lead to Rome? Equifinality set-size and commitment to goals and means. European Journal of Social Psychology, 41, 344-352. https://doi. org/10.1002/ejsp.780.

Lee, S., McClain, C., Webster, N., \& Han, S. (2016). Question order sensitivity of subjective well-being measures: Focus on life satisfaction, self-rated health, and subjective life expectancy in survey instruments. Quality of Life Research, 25, 2497-2510.

Locke, E. A., \& Latham, G. P. (Eds.). (2013). New developments in goal setting and task performance. Routledge:Taylor \& Francis Group.

Locke, E. A., Latham, G. P., \& Erez, M. (1988). The determinants of goal commitment. Academy of Management Review 13, 23-39. https://doi.org/10.2307/258352.

Madrid, J., \& Quick, J. (2007). Get over it and get on with it: Using the ten principles of entelechy to conquer change and create abundance. Bloomington: Authorhouse.

Mazza, J. J., Dexter-Mazza, E. T., Miller, A. L., Rathus, J. H., \& Murphy, H. E. (2016). DBT skills in schools: skills training for emotional problem solving for adolescents. New York: Guilford Publications.

McWhirter, E. H., Hackett, G., \& Bandalos, D. L. (1998). A causal model of the educational plans and career expectations of Mexican American high school girls. Journal of Counseling Psychology, 45, 166-181.

Morris, S. B. (2007). Estimating effect sizes from pretest-posttest-control group. Designs. Organizational Research Methods, 11, 364-386.

Neipp, M. C., Beyebach, M., Nuñez, R. M., \& Martínez-González, M. C. (2016). The effect of solutionfocused versus problem-focused questions: A replication. Journal of marital and family therapy, 42(3), $525-535$.

O’Connell, B., \& Palmer, S. (2008). Solution-focused coaching. In S. Palmer \& A. Whybrow (Eds.), Handbook of coaching psychology: A guide for practitioners (pp. 278-292). New York: Routledge. 
O'Connell, B., Palmer, S., \& Williams, H. (2012). Solution-focused coaching in practice. Hove: Routledge.

Oettingen, G., \& Gollwitzer, P. M. (2010). Strategies of setting and implementing goals: Mental contrasting and implementation intentions. In J. E. Maddux \& J. P. Tangney (Eds.), Social psychological foundations of clinical psychology (pp. 114-135). New York: Guilford Press.

Oettingen, G., Hönig, G., \& Gollwitzer, P. M. (2000). Effective self-regulation of goal attainment. International Journal of Educational Research, 33, 705-732.

Oettingen, G., Mayer, D., Thorpe, J. S., Janetzke, H., \& Lorenz, S. (2005). Turning fantasies about positive and negative futures into self-improvement goals. Motivation and Emotion, 29, 237-267.

Oettingen, G., Pak, H., \& Schnetter, K. (2001). Self-regulation of goal setting: Turning free fantasies about the future into binding goals. Journal of Personality and Social Psychology, 80, 736-753.

Ratner, H., \& Yusuf, D. (2015). Brief coaching with children and young people: A solution-focused approach. New York: Routledge.

Roberson, L. (1990). Prediction of job satisfaction from characteristics of personal work goals. Journal of Organizational Behavior, 11(1), 29-41.

Schnelle, J., Brandstätter, V., \& Knöpfel, A. (2010). The adoption of approach versus avoidance goals: The role of goal-relevant resources. otivation and Emotion, 34, 215-229.

Seligman, M. E. P., \& Csikszentmihalyi, M. (2000). Positive psychology: An introduction. American Psychologist, 55(1), 5-14. https://doi.org/10.1037/0003-066X.55.1.5.

Stobie, I., Boyle, J., \& Woolfson, L. (2005). Solution-focused approaches in the practice of UK educational psychologists: A study of the nature of their application and evidence of their effectiveness. School Psychology International, 26(1), 5-28.

Tang, X., Wang, M.-T., Guo, J., \& Salmela-Aro, K. (2019). Building grit: The longitudinal pathways between mindset, commitment, grit, and academic outcomes. Journal of Youth and Adolescence, 48, 850-863. https://doi.org/10.1007/s10964-019-00998-0.

Taylor, E. R. (2019). Solution-focused therapy with children and adolescents: Creative and play based approaches. NY: Routledge.

Theeboom, T., Beersma, B., \& Van Vianen, A. E. M. (2016). The differential effects of solution-focused and problem-focused coaching questions on the affect, attentional control and cognitive flexibility of undergraduate students experiencing study-related stress. The Journal of Positive Psychology: Dedicated to Furthering Research and Promoting Good Practice, 11, 460-469.

Usher, E. L., \& Pajares, F. (2008). Self-efficacy for self-regulated learning: A validation study. Educational and Psychological Measurement, 68(3), 443-463. https://doi.org/10.1177/0013164407308475.

Wolraich, M. (2008). Developmental-behavioral pediatrics: Evidence and practice. Philadelphia: Mosby/ Elsevier.

Zhang, A., Franklin, C., Currin-Mcculloch, J., Park, S., \& Kim, J. S. (2017). The effectiveness of strengthbased, solution-focused brief therapy in medical settings: A systematic review and meta-analysis of randomized controlled trials. Journal of Behavioral Medicine, 41(2), 139-151.

Zhang, W., \& Gutierrez, O. (2007). Information technology acceptance in the social services sector context: An exploration. Social Work, 52(3), 221-231.

Publisher's Note Springer Nature remains neutral with regard to jurisdictional claims in published maps and institutional affiliations. 